\title{
The crystal structure of $\mathbf{N}$-(2-methoxy-4,5-bis [phenylselanyl]phenyl)picolinamide, $\mathrm{C}_{25} \mathrm{H}_{20} \mathrm{~N}_{2} \mathrm{O}_{2} \mathrm{Se}_{2}$
}
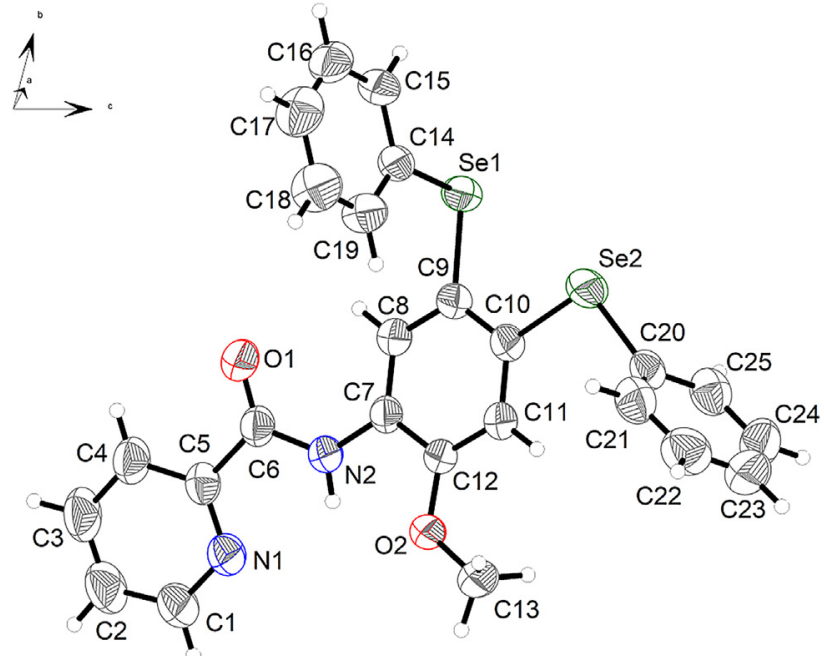

https://doi.org/10.1515/ncrs-2020-0486

Received September 21, 2020; accepted October 13, 2020;

published online November 2, 2020

\begin{abstract}
$\mathrm{C}_{25} \mathrm{H}_{20} \mathrm{~N}_{2} \mathrm{O}_{2} \mathrm{Se}_{2}$, triclinic, $P \overline{1}$ (no. 2), $a=9.6050(2) \AA$, $b=9.7531(2) \AA, c=13.2788(2) \AA, \alpha=75.130(1)^{\circ}, \beta=81.824(1)^{\circ}$, $y=72.329(2)^{\circ}, V=1142.75(4) \AA^{3}, Z=2, R_{g t}(F)=0.0393$, $w R_{\text {ref }}\left(F^{2}\right)=0.1092, T=294 \mathrm{~K}$.
\end{abstract}

CCDC no.: 1969153

The molecular structure is shown in the Figure. Table 1 contains crystallographic data and Table 2 contains the list of the atoms including atomic coordinates and displacement parameters.

*Corresponding author: Lu-Yao Wang, Department of Chemistry, Capital Normal University, Beijing, 100048, China,

E-mail: xdwly@163.com

Qing Wang, Meng-Meng Zhang, Ping-Ping Li and Xiao-Long Guo, Department of Chemistry, Capital Normal University, Beijing, 100048, China. https://orcid.org/0000-0002-1927-6731 (Q. Wang)
Table 1: Data collection and handling.

\begin{tabular}{|c|c|}
\hline Crystal: & Colourless plate \\
\hline Size: & $0.30 \times 0.20 \times 0.10 \mathrm{~mm}$ \\
\hline Wavelength: & Cu $K \alpha$ radiation $(1.54184 \AA$ ) \\
\hline$\mu:$ & $4.24 \mathrm{~mm}^{-1}$ \\
\hline Diffractometer, scan mode: & XtaLAB AFC12 (RINC), $\omega$ \\
\hline$\theta_{\max }$, completeness: & $74.5^{\circ},>99 \%$ \\
\hline $\begin{array}{l}N(h k l)_{\text {measured }}, N(h k l)_{\text {unique }} \\
R_{\text {int }}:\end{array}$ & $12,742,4516,0.028$ \\
\hline Criterion for $I_{\mathrm{obs}}, N\left(h k l_{\mathrm{gt}}\right.$ : & $I_{\text {obs }}>2 \sigma\left(I_{\text {obs }}\right), 4268$ \\
\hline$N(\text { param })_{\text {refined }}$ & 281 \\
\hline Programs: & CrysAlis $^{\text {PRO }}[1]$, Olex2 [2], SHELX $[3,4]$ \\
\hline
\end{tabular}

\section{Source of material}

All reagents and solvents were purchased form commercial sources and used as received. A solution of $N$ (2-methoxyphenyl)picolinamide (0.0456 g; $0.2 \mathrm{mmol})$ in acetonitrile $(1 \mathrm{~mL})$ was added to a mixture of diphenyl diselenide (0.1250 g; $0.4 \mathrm{mmol})$, potassium persulfate (0.0180 g; $0.4 \mathrm{mmol})$ and selectfluor (1-(Chloromethyl)4-fluoro-1,4-diazabicyclo[2.2.2]octane-1,4-diium ditetrafluoroborate; $0.0350 \mathrm{~g} ; 0.1 \mathrm{mmol}$ ) with constant stirring. The mixture was heated to $373.15 \mathrm{~K}$ for $24 \mathrm{~h}$. The acetonitrile was evaporated under vacuum to leave a clear colourless solid. The crude product was purified by column chromatography to give the title compound ( $0.0656 \mathrm{~g}$; $61 \%$ yield) as clear colourless crystals.

\section{Experimental details}

The carbon-bound hydrogen atoms were placed in their geometrically idealized positions and constrained to ride on their parent atoms.

\section{Comment}

In recent years, many groups have studied the pharmacological activities and functions of diaryl selenides. 
Table 2: Fractional atomic coordinates and isotropic or equivalent isotropic displacement parameters $\left(\AA^{2}\right)$.

\begin{tabular}{|c|c|c|c|c|}
\hline Atom & $x$ & $y$ & $z$ & $U_{\text {iso }}{ }^{*} / U_{\text {eq }}$ \\
\hline Se1 & $0.36316(3)$ & $0.81345(3)$ & $0.39410(2)$ & $0.05391(12)$ \\
\hline $\mathrm{Se} 2$ & $0.17160(4)$ & $0.68928(3)$ & $0.60438(3)$ & $0.06672(13)$ \\
\hline 01 & $0.4925(3)$ & $0.4638(2)$ & $0.15025(16)$ & $0.0724(6)$ \\
\hline 02 & $0.3276(2)$ & 0.18640 (19) & $0.47048(14)$ & $0.0557(5)$ \\
\hline N1 & $0.5696(3)$ & $0.0757(3)$ & 0.19757 (19) & $0.0573(6)$ \\
\hline N2 & $0.4670(3)$ & $0.2936(2)$ & $0.29923(16)$ & 0.0489 (5) \\
\hline $\mathrm{H} 2 \mathrm{~N}$ & 0.4832 & 0.2002 & 0.3246 & $0.059^{*}$ \\
\hline $\mathrm{C} 1$ & $0.6210(4)$ & $-0.0346(4)$ & $0.1482(3)$ & $0.0696(8)$ \\
\hline $\mathrm{H} 1$ & 0.6264 & -0.1303 & 0.1862 & $0.083^{*}$ \\
\hline $\mathrm{C} 2$ & $0.6666(4)$ & $-0.0138(4)$ & $0.0437(3)$ & 0.0809 (10) \\
\hline $\mathrm{H} 2$ & 0.7035 & -0.0939 & 0.0125 & $0.097^{\star}$ \\
\hline $\mathrm{C} 3$ & $0.6566(4)$ & $0.1266(4)$ & $-0.0132(3)$ & $0.0792(10)$ \\
\hline H3 & 0.6855 & 0.1436 & -0.0841 & $0.095^{\star}$ \\
\hline $\mathrm{C} 4$ & $0.6029(4)$ & $0.2432(4)$ & $0.0358(2)$ & 0.0649 (8) \\
\hline $\mathrm{H} 4$ & 0.5950 & 0.3399 & -0.0011 & $0.078^{*}$ \\
\hline $\mathrm{C} 5$ & $0.5613(3)$ & $0.2117(3)$ & $0.1412(2)$ & $0.0500(6)$ \\
\hline $\mathrm{C} 6$ & $0.5040(3)$ & $0.3361(3)$ & 0.19668 (19) & $0.0502(6)$ \\
\hline $\mathrm{C} 7$ & $0.4047(3)$ & $0.3860(3)$ & $0.36927(18)$ & $0.0440(5)$ \\
\hline $\mathrm{C} 8$ & $0.4132(3)$ & $0.5296(3)$ & $0.35226(19)$ & $0.0466(5)$ \\
\hline $\mathrm{H} 8$ & 0.4651 & 0.5682 & 0.2927 & $0.056^{\star}$ \\
\hline C9 & $0.3454(3)$ & $0.6165(3)$ & 0.42301 (19) & $0.0464(5)$ \\
\hline C10 & $0.2681(3)$ & $96(3)$ & 82 (19) & $0.0470(5)$ \\
\hline C11 & $0.2620(3)$ & 4140 (3) & 0.53178 (19) & $0.0481(5)$ \\
\hline $\mathrm{H} 11$ & 0.2127 & 0.3746 & 0.5925 & $0.058^{*}$ \\
\hline C12 & $0.3288(3)$ & $0.3283(3)$ & $0.46073(18)$ & 0.0447 (5) \\
\hline $\mathrm{C} 13$ & $0.2467(5)$ & $0.1209(4)$ & $0.5584(2)$ & $0.0740(10)$ \\
\hline $\mathrm{H} 13 \mathrm{a}$ & 0.2521 & 0.0227 & 0.5540 & $0.111^{*}$ \\
\hline $\mathrm{H} 13 \mathrm{~b}$ & 0.1462 & 0.1792 & 0.5589 & $0.111^{*}$ \\
\hline $\mathrm{H} 13 \mathrm{c}$ & 0.2873 & 169 & 215 & $0.111^{\star}$ \\
\hline C14 & $0.2068(3)$ & $0.9151(3)$ & $0.30326(19)$ & $0.0470(5)$ \\
\hline $\mathrm{C} 15$ & $0.1971(3)$ & $1.0610(3)$ & $0.2539(2)$ & $0.0574(7)$ \\
\hline $\mathrm{H} 15$ & 0.2634 & 1.1062 & 0.2662 & 0.069 * \\
\hline $\mathrm{C} 16$ & $0.0879(4)$ & $1390(3)$ & $1861(3)$ & $0.0677(8)$ \\
\hline H16 & 0.0810 & 1.2371 & 0.1531 & $0.081^{*}$ \\
\hline C17 & $-0.0097(4)$ & $0731(4)$ & $0.1671(3)$ & $0.0757(9)$ \\
\hline $\mathrm{H} 17$ & -0.0830 & 1.1261 & .1218 & $0.091^{*}$ \\
\hline C18 & $0.0014(4)$ & $0.9269(4)$ & $0.2160(3)$ & $0.0768(9)$ \\
\hline $\mathrm{H} 18$ & -0.0641 & 0.8813 & .2029 & $0.092 *$ \\
\hline C19 & $0.1087(3)$ & $0.8486(3)$ & $0.2838(2)$ & $0.0606(7)$ \\
\hline H19 & 0.1151 & 0.7507 & 0.3167 & $0.073^{*}$ \\
\hline $\mathrm{C} 20$ & $0.1005(3)$ & $5560(3)$ & $7181(2)$ & $0.0520(6)$ \\
\hline $\mathrm{C} 21$ & $-0.0362(4)$ & $0.5370(4)$ & $0.7191(3)$ & $0.0672(8)$ \\
\hline $\mathrm{H} 21$ & -0.0895 & 0.5779 & 0.6601 & $0.081^{*}$ \\
\hline $\mathrm{C} 22$ & $-0.0940(4)$ & $0.4567(5)$ & $0.8082(3)$ & 0.0805 (10) \\
\hline $\mathrm{H} 22$ & -0.1866 & 0.4447 & 0.8088 & $0.097^{*}$ \\
\hline $\mathrm{C} 23$ & $-0.0169(5)$ & $0.3957(4)$ & $0.8942(3)$ & $0.0843(11)$ \\
\hline $\mathrm{H} 23$ & -0.0566 & 0.3423 & 0.9537 & $0.101^{*}$ \\
\hline $\mathrm{C} 24$ & $0.1197(5)$ & $0.4128(4)$ & $0.8933(3)$ & 0.0801 (10) \\
\hline $\mathrm{H} 24$ & 0.1728 & 0.3702 & 0.9524 & $0.096^{*}$ \\
\hline $\mathrm{C} 25$ & $0.1790(3)$ & $0.4925(4)$ & 0.8059 (3) & $0.0640(7)$ \\
\hline $\mathrm{H} 25$ & 0.2718 & 0.5036 & 0.8059 & $0.077^{*}$ \\
\hline
\end{tabular}

Various diaryl selenides have shown good antiproliferation, antimicrobial, antiinflammatory, antihypertensive, and antiviral activities, and even had been used as scaffolds in organic synthesis and catalysis [4, 5]. Thus, it is necessary to investigate further examples of diaryl selenides.

The title compound contains one $N$-(2-methoxy-4,5-bis [phenylselanyl]phenyl) picolinamide molecule. There is a $\mathrm{N}$-(2-methoxyphenyl) picolinamide ring and two selenophenyl groups in the structure. The nitrogen atom in $\mathrm{N}$ (2-methoxyphenyl) picolinamide ring and the selenium atom in two selenobenzenes were coplanar, and the dihedral angles of the central aryl ring plane and the two planes defined by the selenophenyl groups were $84.49(6)^{\circ}$ and 83.76(9) ${ }^{\circ}$, respectively. The Se-C distances are 1.916(3) $\AA$ and 1.922(2) A, respectively.

Author contribution: All the authors have accepted responsibility for the entire content of this submitted manuscript and approved submission.

Research funding: We are very grateful for the financial assistance provided by Capital Normal University.

Conflict of interest statement: The authors declare no conflicts of interest regarding this article.

\section{References}

1. Agilent Technologies. CrysAlis ${ }^{P R O}$ Software System, Version. 171.39.33c; Agilent Technologies UK Ltd: Oxford, UK, 2018.

2. Dolomanov O. V., Bourhis L. J., Gildea R. J., Howard J. A. K., Puschmann H. OLEX2: a complete structure solution, refinement and analysis program. J. Appl. Crystallogr. 2009, 42, 339-341.

3. Sheldrick G. M. Crystal structure refinement with SHELXL. Acta Crystallogr. 2015, C71, 3-8.

4. Sheldrick G. M. SHELXT - integrated space-group and crystalstructure determination. Acta Crystallogr. 2015, A71, 3-8.

5. Luo D., Wu G., Yang H. Copper-catalyzed three-component reaction for regioselective aryl- and heteroarylselenation of indoles using selenium powder. J. Org. Chem. 2016, 81, 4485-4493. 